\title{
Enseñar y aprender en la virtualidad
}

\author{
Albert Sangrà \\ Universitat Oberta de Catalunya \\ asangra@campus.uoc.es
}

\section{Resumen}

La educación en la virtualidad no se sitúa necesariamente en ninguna orientación educativa concreta. La diferencia más importante entre la educación en la presencialidad y en la virtualidad reside en el cambio de medio y en el potencial educativo que se deriva de la optimización del uso de cada medio. El artículo establece que los elementos diferenciales de los procesos educativos en ambientes virtuales deben ser de dos tipos: metodológicos y organizativos.

A partir de la experiencia en la UOC, analiza los cambios vinculados a la docencia virtual que se están sucediendo y que afectan a los propios docentes, a los estudiantes y a la cultura organizativa de las instituciones. Plantea si estamos ante la emergencia de un nuevo paradigma, basado en el aprendizaje en red y en el uso de Internet en la educación y concluye contrastando la formación virtual con el reto de la globalidad que se plantea la sociedad actual.

Palabras clave: virtualidad, aprendizaje, docencia, modelos metodológicos, modelos organizativos, tecnologías de la información y la comunicación.

\section{Resum}

L'educació en la virtualitat no es situa necessàriament en cap orientació educativa concreta. La diferència més important entre l'educació en la presencialitat i en la virtualitat resideix en el canvi de medi i en el potencial educatiu que se'n deriva de l'optimització de l'ús de cada mitjà. L'article estableix que els elements diferencials dels processos educatius en ambients virtuals han de ser de dos tipus: metodològics i organitzatius.

A partir de l'experiència en la UOC, analitza els canvis vinculats a la docència virtual que s'estan succeint i que afecten als propis docents, als estudiants i a la cultura organitzativa de les institucions. Planteja si ens trobem davant l'emergència d'un nou paradigma, basat en l'aprenentatge en xarxa i en l'ús d'Internet en l'educació i conclou contrastant la formació virtual amb el repte de la globalitat que es planteja la societat actual.

Paraules clau: virtualitat, aprenentatge, docència, models metodològics, models organitzatius, tecnologies de la informació i la comunicació.

\section{Abstract}

Education in virtual environments is not necessarily located at any special educational approach. The most important difference between it and face-to-face education is that we change the environment where or through we teach and students learn, and the 
educational potential related to the best use of each environment, This paper focuses on the two main elements that make the difference in the educational processes in virtual environments: methodology and organisation.

From the experience in the UOC, changes related to virtual teaching and learning and organisational culture are analysed. It asks if we are in front of the emergence of a new paradigm, based on networked learning and the use of the Internet in education, and concludes facing virtual teaching and learning with the challenge of globalisation of the society.

Key words: virtuality, learning, teaching, methodological models, organisational models, information and communication technologies.

\section{Sumario}

La virtualidad, un medio distinto

¿Cómo educar en la virtualidad?

La experiencia de la UOC

Epílogo: la docencia virtual

como respuesta al reto de la globalidad

Bibliografía

La docencia virtual: ¿̨resultado

de la evolución de la enseñanza

a distancia o nuevo paradigma educativo?

\section{La virtualidad, un medio distinto}

La educación en la virtualidad, es decir, desde la no-presencia en entornos virtuales de aprendizaje, no se sitúa necesariamente en ninguna orientación educativa concreta. Al igual que en la presencialidad existe la convivencia entre orientaciones y didácticas diversas, siempre que éstas actúen de forma coherente con las finalidades educativas y con los fines de la educación, de la misma forma sucede en la virtualidad. El aprendizaje en ambientes virtuales es el resultado de un proceso, tal y como valoraríamos desde la perspectiva humanista, en el que el alumno construye su aprendizaje. También puede ser el producto realizado a partir de la práctica, como puede ser el caso del trabajo a partir de simuladores. Y evidentemente la acción resultante de un trabajo de análisis crítico. Es decir, que de la misma forma que la presencialidad permite diferentes perspectivas de análisis o de valoración de la educación, éstas también son posibles en la virtualidad.

La diferencia más importante entre la educación en la presencialidad y en la virtualidad reside en el cambio de medio y en el potencial educativo que se deriva de la optimización del uso de cada medio. No podemos hacer lo mismo en medios distintos, aunque nuestras finalidades educativas y, por tanto, los resultados que perseguimos sean los mismos, pero debemos saber de antemano que el camino que debemos recorrer es distinto. En la aceptación de esta diferencia de medio de comunicación reside el éxito o el fracaso de la actividad educativa.

En este sentido cabe la reflexión sobre el hecho que los modelos virtuales no tendrán éxito si se basan en intentar replicar los modelos presenciales. La clase 
magistral es una clase presencial, y suponiendo que sea un buen recurso, que a veces lo será, no puede "copiarse» en otro medio. Será necesaria una adaptación, que aproveche lo mejor que ese medio ofrece y que, de esta forma, alcance los mismos objetivos formativos que se plantearía una acción presencial $^{1}$.

Educación y virtualidad se complementan en la medida en que la educación puede gozar de las posibilidades de creatividad de la virtualidad para mejorar o diversificar sus procesos y acciones encaminados a la enseñanza y al aprendizaje, mientras que la virtualidad como sistema se beneficia de la metodología de trabajo educativo y de comunicación, necesaria en aquellos casos habituales en los que la finalidad de la relación en la red sobrepasa la de la búsqueda de información.

\section{¿Cómo educar en la virtualidad? La experiencia de la UOC}

Existen caminos diversos para llegar al mismo punto final: la educación de la persona. En la virtualidad, al igual que en la presencialidad, los distintos caminos conviven y se complementan. Las actuaciones educativas en las que tanto el educador como el educando sepan ser flexibles en el proceso y adaptar las metodologías y las didácticas a las necesidades educativas, al perfil de los componentes del proceso y al contexto de aprendizaje tienen una posibilidad de éxito bastante garantizada, así como un alto nivel de calidad formativa. Pero en la virtualidad deben cumplirse unos mínimos ya que la gran diferencia entre la presencialidad y la no presencialidad reside en que ésta necesita de la explicitación de los procesos y de las metodologías de aprendizaje, más que la presencialidad, y por ello la tarea debe estar siempre previamente diseñada y debe ser conocida por el estudiante.

¿Cuáles deberían ser los elementos a tener en cuenta como diferenciales en los procesos educativos en ambientes virtuales? Consideramos que deben ser de dos tipos: metodológicos y organizativos.

\section{Metodología para la educación en la virtualidad}

Sin perjuicio de intentar implantar un modelo metodológico equilibrado (Sangrà y Duart, 2000), la metodología educativa para entornos virtuales de aprendizaje debe estar centrada en el estudiante. No puede ser de otro modo,

1. En definitiva el proceso de aprendizaje, así como la educación, es un planteamiento global que tiene concreciones diversas según el medio a través del cual se vehicula. Actualmente, gracias a las nuevas tecnologías de la información aplicadas a la educación, podemos comprobar hasta qué punto el replanteamiento pedagógico que hacemos del uso educativo de los espacios de no presencialidad o asíncronos es el mismo que para los espacios convencionales presenciales de educación y aprendizaje. En este sentido, es interesante la aportación de Alain Tait y Roger Mills (eds.). The convergence of distance and conventional education. Londres: Routledge, 1999. 
sobre todo teniendo en cuenta las características especiales de los estudiantes no presenciales, entre las cuales destacamos que son personas de más de 25 años de edad con trabajo estable y para las cuales el problema no es la distancia ya que no viven aisladas, sino el tiempo, es decir, la imposibilidad de estudiar o acceder a los centros de formación convencionales en horarios preestablecidos. Necesitan de un sistema que se adapte a ellos, no ellos al sistema.

La UOC, universidad pionera en el mundo en el uso completo de Internet para la formación superior homologada, ha desarrollado su modelo educativo a partir de las necesidades de los estudiantes. De ahí que su oferta haya crecido de forma exponencial en cinco años, de 200 alumnos en el curso piloto en el año 1995 hasta los más de 15.000 de la actualidad en 11 titulaciones homologadas. La clave del éxito de la UOC reside, sobre todo, en haber comprendido desde el inicio que para organizar la educación en la virtualidad es necesario disponer de un modelo educativo basado en la asincronía que permite dar respuesta a las necesidades formativas de muchas personas en nuestra sociedad actual, así como dotarse de una organización flexible pensada para la virtualidad. Replicar el modelo clásico de organización universitaria hubiera llevado al fracaso el proyecto de la UOC.

La UOC se presenta como una oportunidad para miles de personas adultas que trabajan y que necesitan una formación constante para adaptarse a los cambios constantes. Y esa formación debe llevarse a cabo a partir del conocimiento de las necesidades de las personas y de las organizaciones. No se trata de abrir las puertas de la universidad, se trata de llevar la universidad a casa de cada estudiante.

El modelo de la UOC, centrado en el estudiante, se basa en cuatro pilares:

— La flexibilidad, en respuesta a la adaptación a las necesidades diversas de un estudiante diverso. El perfil de los estudiantes de la UOC corresponde al de una persona que necesita de un sistema que pueda adaptarse a su realidad personal, profesional y familiar. El modelo pedagógico de la UOC permite flexibilidad en el modelo de evaluación, en el Plan de Trabajo de las asignaturas, en el acceso a las fuentes de información, etc. Todo está pensado para satisfacer a unos estudiantes que necesitan de un sistema que se adapte a sus necesidades y no al revés.

- La cooperación, ya que los estudiantes no están solos en su proceso de aprendizaje. En el Campus Virtual de la UOC es posible cooperar con los demás estudiantes realizando trabajos en equipo, o con los consultores, o con personas diversas de la comunidad universitaria en la creación de grupos de interés.

- La personalización, que facilita el trato individual de las necesidades formativas de cada estudiante. El sistema de evaluación progresiva de la UOC es una manifestación de trato personalizado en el proceso de aprendizaje en el que tanto los materiales multimedia de aprendizaje como los consultores tratan de forma personal al estudiante en sus necesidades formativas. 
- La interactividad, evidente en el entorno virtual de aprendizaje que llamamos Campus Virtual, es la base en la que se establecen las relaciones formativas en la UOC. La interacción es múltiple ya que no sólo abarca la acción docente sino que además se establece entre estudiantes y entre estos con la propia universidad.

A parte del modelo pedagógico general, que debe dar coherencia a la acción educativa, debemos trabajar en metodologías concretas de aprendizaje, o mejor todavía, en la adaptación de las metodologías convencionales de aprendizaje a los entornos virtuales. Métodos como el del caso, o los debates, o las exposiciones en clase, los mapas conceptuales, etc., son fácilmente transportables a un espacio virtual; únicamente debemos tener en cuenta que la secuencia didáctica de elaboración y de implementación es distinta, y en algunos casos más dilatada en el tiempo ${ }^{2}$.

\section{Organizar la educación en la virtualidad}

La organización (institución formativa) debe poner las posibilidades de las nuevas tecnologías de la información y de la comunicación al servicio del estudiante, es decir, al proceso de aprendizaje. La tecnología será un medio, un valor añadido, pero no una finalidad en sí misma. Los entornos virtuales de aprendizaje deben ser el principal espacio de comunicación entre la comunidad virtual que forma la organización o institución de formación. Estos espacios deben permitir la relación de los estudiantes entre ellos, de los estudiantes con los profesores y de los profesores entre sí, así como de cualquier miembro de la comunidad con la organización y viceversa.

Los entornos virtuales de aprendizaje son el lugar en el que se encuentran las materias de estudio, así como los materiales de aprendizaje. Los estudiantes, los profesores, etc., forman todos parte de la comunidad. Cada materia de formación debe disponer de una serie de posibilidades de trabajo: debates, foros, mensajes electrónicos, actividades, enlaces, etc. Los materiales de aprendizaje, como ya hemos observado, deberán permitir la interacción y la construcción colectiva del conocimiento.

La organización para la educación en la virtualidad necesita de una estructura particular. Al igual que cualquier otra organización educativa, las organizaciones educativas virtuales deben gestionar tanto los procesos que afectan a los estudiantes (gestión académica) como los que afectan a la docencia (gestión docente). Pero la forma de hacerlo será diferente. Además, las organiza-

2. La UOC ha desarrollado un instrumento de apoyo llamado Asistente de Recursos Metodológicos (ARM), que pone a disposición de todos sus profesores. La finalidad de este aplicativo es ayudar a los docentes en su tarea de implementación de recursos metodológicos para la docencia. El asistente se fundamenta en el diseño educativo de los recursos, aunque también incorpora plantillas tecnológicas para su fácil incorporación al entorno virtual. 
ciones no presenciales, según cual sea su modelo pedagógico, deberán gestionar también la producción o edición de materiales educativos.

La gestión de las organizaciones educativas virtuales deberá actuar en función de los siguientes parámetros:

- No presencialidad. La organización virtual actuará en el ámbito de la asincronía, es decir, de la no-coincidencia en el espacio ni en el tiempo. Esto condiciona, sin duda, su modelo organizativo.

- Transversalidad. Es importante que exista un nivel de transversalidad en la gestión de cualquier organización virtual. La virtualidad facilita los procesos transversales y los optimiza. Lo que es válido para una materia o curso, para un grupo de personas, puede ser válido también para otros muchos. Tener en cuenta este principio y trabajarlo de forma coherente con el modelo educativo ayuda a homogeneizar o a armonizar todos los procesos de gestión, tanto académica como docente. Esta transversalidad, además, favorece el trabajo multidisciplinar entre las diferentes áreas o ámbitos de actuación.

- Globalidad. Los procesos de gestión deben actuar de forma sistémica en el marco de la organización. Los procesos deben ser coherentes entre sí y de esta forma garantizar la cohesión organizativa de toda la universidad u organización. No se gestiona únicamente desde una perspectiva (estudiante, profesor, organización, etc.) si no que la organización virtual permite y favorece los procesos de gestión global, desde todas las perspectivas. Un mismo proceso organizativo puede — debe- ser afrontado desde diversas perspectivas según quienes sean sus usuarios, pero el resultado será siempre un proceso global. Pero volveremos sobre este tema al final.

Está claro que no existe una única forma de organizar una institución educativa, ni convencional ni virtual, pero sí que parece evidente que el modelo organizativo que se adopte para la gestión y la administración tiene claras consecuencias en el funcionamiento de la organización y, por tanto, en el facilitar o no el logro de sus objetivos. De ahí la importancia de organizar a partir del modelo educativo, es decir, de situar la gestión al servicio de la aplicación del sistema educativo.

\section{La docencia virtual: ¿̨resultado de la evolución de la enseñanza a distancia o nuevo paradigma educativo?}

Con el objetivo de hacer llegar la educación a todo aquel que la necesita, aparecieron las prácticas de educación a distancia. Estas prácticas han exigido siempre la existencia de un elemento mediador entre el docente y el discente. Generalmente, este mediador ha sido una tecnología, que ha ido variando en cada momento. Si históricamente nos referíamos al correo convencional, que establecía una relación epistolar entre el profesor y el estudiante, con el tiempo hemos ido introduciendo nuevas tecnologías que, por su coste y su accesibilidad, nos permiten evolucionar en esta relación a distancia (Bates, 1995). 
Si bien Wedemeyer (1981) expresa sus dudas respecto al hecho de que exista una verdadera teoría de la educación a distancia, también es cierto que ha habido quien ha intentado demostrar lo contrario: que ésta, o éstas, existen.

Basándonos en este concepto, observaremos que existe un cierto acuerdo para establecer tres grandes bloques de teorías o, por lo menos, de intentos de teorizar la base de la educación a distancia (Keegan, 1996):

a) Teorías basadas en la autonomía y la independencia del estudiante (Delling, Wedemeyer y Moore).

b) Teoría basada en el proceso de industrialización de la educación (Peters).

c) Teorías basadas en la interacción y la comunicación (Baath, Holberg, Sewart y otros).

Hace relativamente poco, Simonson, Schlosser y Hanson (1999) nos permiten analizar la comparación entre algunas de éstas, a la vez que nos ofrecen su visión particular.

Sin embargo, nos acojamos a la teoría que nos acojamos, siempre aparecerán unos elementos en juego comunes en todos los casos. El primero de éstos es el objeto de cualquier sistema educativo: el estudiante. El análisis de sus necesidades y de sus características específicas (edad, nivel educativo previo, estatus social, disponibilidad de tiempo para el estudio, etc.) se convierten en elementos absolutamente condicionantes que, en caso de no tenerlos en cuenta, impiden definir cualquier modelo de educación a distancia mediado por alguna tecnología. De hecho, hay quien objetaría que esto podría aplicarse a la educación en general, y así es. Sin embargo, así como en la formación presencial o convencional (Tait y Mills, 1999), por regla general, nos dirigimos a un grupo, sea éste homogéneo o no, cuando entramos en contextos de educación a distancia el individuo ha sido analizado de forma segregada con respecto a su grupo de origen, si lo hay.

Un segundo elemento es el docente. Es fundamental el papel que el profesor desarrolla en la relación con el estudiante. En realidad, existe un hecho muy interesante en las teorías más comúnmente analizadas: todas hablan de «diálogo", o de un concepto equivalente, como un modelo de educación a distancia. El concepto «diálogo» nos aporta elementos muy enriquecedores en ciertos casos, pero también hay ocasiones en que no nos aporta prácticamente nada. Sólo Peters, que además mantiene que no intenta elaborar una teoría de la educación a distancia (Peters, 1989), no lo utiliza.

El tercer elemento son los recursos que se ponen a disposición de los estudiantes para el aprendizaje. Y ahí es donde aparece otro de los conceptos básicos: la interacción. Hablemos de modelos basados en la autonomía o de modelos basados en la comunicación; en ambos casos observamos que la interacción es considerada un efecto positivo. Se han analizado las diferentes tipologías de interacción más habituales en las relaciones que se establecen en los modelos de educación a distancia, llegándose a plantear modelos transaccionales (Moore, 1989), pero siempre se ha realizado este análisis en un contexto donde la comunicación entre estudiantes y profesores era posible, pero no lo era 
entre los propios estudiantes si no «rompían» con la distancia desde una perspectiva física.

Desde una concepción de educación basada en la idea de que el contacto entre el educador y el estudiante es la única actividad fundamental que posibilita la educación, de base oral, con un uso escaso de la lengua escrita; pasando por la invención de la imprenta, que incluía un tercer elemento, los libros, en la relación educacional, desde estas concepciones, decimos, estamos llegando a un nuevo concepto en el que el equipo y el trabajo en red se valoran mucho, que refleja los cambios sociales y la fuerza de trabajo (Harasim et al., 1995): el aprendizaje en red.

Las redes tecnológicas permiten la interacción entre estudiantes, expertos y fuentes de información para acumular conocimiento de manera progresiva y, así, desarrollar habilidades. Los atributos del trabajo en red hacen hincapié en las oportunidades y recursos disponibles para los estudiantes y los profesores. Éstos no están limitados a causa de su situación geográfica: es fácil llegar a los expertos, ya que tenemos acceso a las mejores bibliotecas y bases de datos en el mundo.

La mayor parte de los aspectos que se aprenden por las redes no se puede alcanzar en una clase tradicional. No es posible que cada miembro de un grupo participe activamente y con frecuencia en una clase tradicional con límites de tiempo.

Un excelente ejemplo en este sentido son las redes de aprendizaje. Muchos de los aspectos tratados en el trabajo de estas redes son nuevos y no se pueden alcanzar en sistemas escolares clásicos. A menudo, la oportunidad que tienen varios miembros de un colectivo de participar activamente y con frecuencia no es posible en un sistema cara a cara, que depende en gran medida de la coincidencia de espacio y tiempo. Las nuevas oportunidades que caracterizan estas redes nos permiten pensar en mejoras para asumir un nivel más elevado de conocimiento y en las posibilidades de la interacción social.

Desde un punto de vista exclusivamente educacional, la contribución de estas redes es muy valiosa, puesto que ofrece al estudiante muchas más posibilidades de interacción y de mostrarse participativo que al estudiante tradicional cuando está en clase. El trabajo en red les permite aprender no sólo del profesorado, sino también de sus propios compañeros de clase, con quienes puede mantener una relación mucho más cercana. Al mismo tiempo, estos métodos permiten al profesorado actuar desde una perspectiva mucho más interdisciplinaria e integrada. Sin embargo, ¿podríamos decir que este concepto de aprendizaje cooperativo, que actualmente emerge con dificultades, lo hace como un nuevo paradigma educacional?

\section{¿Qué cambios se están desencadenando?}

La organización del tiempo y el espacio en el proceso de aprendizaje necesita cambiar de acuerdo con las inmensas posibilidades de comunicación e información que tenemos si utilizamos herramientas telemáticas en este entorno. 
El profesorado puede organizar el tiempo docente sabiendo que el uso del ordenador también facilita el aprendizaje, que deberá venir guiado por su experiencia. Cuando se usan las posibilidades ofrecidas por estas tecnologías, el profesorado también puede estructurar el espacio y facilitar el trabajo de grupo.

Uno de los cambios, si no el más importante, sí el más complejo de ser asumido por la profesión docente, es el cambio del rol del profesorado. Un rol dedicado a facilitar el aprendizaje, para el que va a ser necesario un perfil diferente y una formación inicial y permanente distinta a la ofrecida hasta el momento y también la asunción de una cultura distinta.

\section{Facilitar el aprendizaje}

Los elementos mencionados con anterioridad harán que el estudiante no sólo se centre en el profesorado, sino también en su proceso de aprendizaje, de manera que consiga avanzar en su tarea según el proceso. Esta descentralización implica facilitar al estudiante las herramientas que necesita, en lugar de dirigirlo en todo momento; estas herramientas estarán cada vez más cercanas al mundo profesional específico al que el estudiante quiere acceder en el futuro.

El profesorado deja de ser la fuente de información para convertirse en el facilitador del aprendizaje. Esto no significa que ahora se limiten a dirigir el proceso de aprendizaje. Por medio de la orientación y la inducción, el objetivo de la acción del profesor es el de proporcionar al estudiante los instrumentos y pistas que lo ayudarán a desarrollar su propio proceso de aprendizaje $y$, al mismo tiempo, atender sus dudas y necesidades.

La evolución de las nuevas tecnologías y el siempre fácil acceso a las fuentes de información está cambiando el papel de los profesores. Maniobrar y moverse en un volumen de información como éste - como hemos dicho antes- se ha convertido en una búsqueda del éxito en un gran laberinto. El riesgo de la pérdida de tiempo en búsquedas inútiles es muy elevado, de modo que debemos aprender a diferenciar lo bueno de lo malo. Necesitamos un filtro adecuado y éste será, por tanto, uno de los papeles del profesor y el formador.

A pesar de ello, es curioso, y a pesar del hecho de que muchos todavía quieren creer que el valor añadido del profesor reside principalmente en lo que sabe - es decir, los contenidos-, el futuro nos muestra que lo más importante no es esto, sino el método: la forma como nosotros, los profesores, hacemos las cosas, cómo proporcionamos a los estudiantes los instrumentos que necesitan para crecer, para encontrar la información —el conocimiento-que los hará capaces de distinguir entre información verdadera y falsa, y cómo les inculcamos un sentido crítico. La manera de conseguir que las personas aprendan será lo que nos distinguirá, lo que nos hará mejores y nos dará prestigio.

De hecho, la realidad ya está superando algunos de estos tópicos. Actualmente, en Internet podemos encontrar iniciativas de profesores privados en línea especializados a quienes los estudiantes pueden preguntar directamente desde casa. No hay horarios ni desplazamientos. A pesar de que la idea no es sustituir al profesor tradicional y el sistema escolar, sí que intenta ofrecer al estu- 
diante un complemento que le pueda dar seguridad en su proceso de aprendizaje.

El debate sobre el adelanto que este tipo de servicio implica está aquí. Sin ningún tipo de duda, algún profesor lo verá sólo como una simple clase privada sofisticada. Otros profesores se darán cuenta del poder de motivar y ofrecer servicios que se pueden realizar por Internet, especialmente para el estudiante de zonas rurales, en hospitales, etc. (con la democratización que implica el hecho de facilitar el acceso a información a grupos que hasta ahora tienen dificultades).

\section{Un perfil necesario}

Las preguntas y las nuevas situaciones, como las descritas anteriormente, como mínimo exponen la incertidumbre y dudas que genera la incorporación de las TIC en la clase. Ahora bien, ¿qué perfil de profesorado requiere esta incorporación? Probablemente, el profesorado debería ser (Sangrà, Bellot e Hinojosa, 2000):

- Más colaborador que solitario: tendrá que trabajar en una red, ya que las posibilidades comunicativas de Internet hacen viable el trabajo interactivo con profesores de centros diferentes.

- Tendría que fomentar/promocionar la participación: esto no sólo significa participación en clase, sino también la corresponsabilidad de los proyectos iniciados en el aula.

- Tendría que reconocer/aceptar el hecho de que ya no tiene la posesión del conocimiento puesto que, por un lado, los estudiantes podrían ser iguales de buenos y competentes, o incluso más, con las TIC y, por el otro, pueden tener acceso a la información de una forma tan fácil como el profesorado.

- Con habilidades organizativas importantes, dado que la clase y la información no serán bidireccionales (del profesorado a los estudiantes y a la inversa), sino que el trabajo con los estudiantes se podrá llevar a cabo en torno a diferentes enfoques de trabajo que provocarán cambios en la manera de enseñar.

- Abierto a la experimentación: abierto a nuevas formas de trabajo tanto con estudiantes como con servicios y productos nuevos que Internet ofrece.

- Con la capacidad/habilidad de modificar, desde el principio hasta el final, la metodología aplicada a la enseñanza del proceso de aprendizaje.

No obstante, lejos de pretender que el cambio es exclusivo para el profesorado, el estudiantado también deberá aprender a modificar su actitud y el rol que ha desarrollado hasta ahora.

\section{El estudiante: de receptor pasivo a protagonista del proceso}

El estudiante también se entrega a los cambios y adopta un papel activo, ya que es el protagonista real de su proceso de aprendizaje, mientras que el edu- 
cador, el profesor, como ya hemos dicho, cambia su función y se convierte en el dinamizador, el guía, el encargado de facilitar el proceso de aprendizaje del estudiante.

De todos modos, el elemento clave del cambio en el proceso educacional es el contenido de lo que se enseña y de cómo se transmite. Mientras que en el paradigma utilizado hasta el momento el contenido se encontraba en el propio conocimiento y experiencia del profesorado, así como en las fuentes estáticas de información (libros, enciclopedias, etc.), en este posible nuevo paradigma, basado en el uso de la tecnología de la información, los contenidos están en una red, y las relaciones comunicativas, también (Harasim et al., 1995). Esto significa que hay un espacio de acceso compartido a partir del cual, y según las habilidades y posibilidades de aquellos que tienen acceso a los medios y soportes disponibles —el profesor, con su experiencia-, el estudiante desarrolla su proceso de aprendizaje.

En el mundo de la educación consideramos que el reto consiste en utilizar la tecnología y las funciones designadas para repartir información e interconectar personas e instituciones como herramienta que facilita el aprendizaje. Sin embargo, la información por la información no es educacional. El acceso a la información y a su tratamiento no implica una acción educacional. Debemos tener en cuenta las variables que forman los procesos de aprendizaje y los sustituyen en función del nuevo contexto en que se desarrollan o tienen lugar. En resumen, necesitamos definir y aplicar un método de enseñanza y aprendizaje concreto específico para este nuevo contexto.

La formación académica, si tiene el fin de ser eficiente y afrontar las necesidades de la sociedad actual, debe promocionar el uso de las nuevas tecnologías de manera instrumental y también estructural, como herramientas que pueden promover y motivar el aprendizaje. El objetivo de la formación inicial de los ciudadanos de este nuevo siglo debe incluir, necesariamente, además del conocimiento especializado, la formación básica en el uso y explotación de las nuevas tecnologías.

Los estudiantes del futuro - un futuro ya muy cercano- tendrán acceso a la información formal e informal cuando quieran, allí donde estén. Serán participantes muy activos. Con ello, el concepto de quién es el profesor y de quién es el estudiante aparecerá muy difuso (Landow, 1997).

\section{Un cambio cultural}

Por último, aunque no se pretende aquí poner ningún límite a los cambios que serán posiblemente necesarios, es importante enfatizar el aspecto del cambio cultural. Si bien es cierto que para aquellas instituciones que vehiculan su oferta formativa de manera completamente virtual, como es el caso de la UOC en España y de otras universidades en el mundo, es relativamente más sencillo que profesores y estudiantes asuman estos nuevos roles y creen una nueva cultura de trabajo en red y en la red; esto puede convertirse en un punto débil en las instituciones presenciales donde las prácticas virtuales son parciales o complementarias. 
Algunas experiencias muy cercanas nos han hecho llegar a esta conclusión. En Cataluña, la UOC y la UAB desarrollan un proyecto de colaboración conjunta llamado Metacampus, donde estudiantes de ambas universidades cursan asignaturas en el entorno virtual de la otra universidad. Igualmente, la Consejería de Universidades, Investigación y Sociedad de la Información impulsó un proyecto de intercambio de asignaturas virtuales entre todas las universidades catalanas, que recibió por nombre Intercampus ${ }^{3}$.

En ambos proyectos se ha podido comprobar como el comportamiento cultural de los estudiantes ante las asignaturas virtuales es distinto si cursan sus estudios en una universidad virtual (en este caso, la UOC) o en una universidad presencial. La frecuencia de conexión, de consulta de los mensajes de correo electrónico, de interacción con el profesor y con los compañeros es mucho menor en el caso de estudiantes que están acostumbrados a asistir físicamente a las clases. Estos perciben las asignaturas virtuales como un añadido con menor valor en sí mismo que las clases presenciales tradicionales.

Igualmente, los profesores que dictan la misma asignatura presencialmente y virtualmente cometen lapsus cuando creen que han establecido determinadas condiciones en su aula virtual, y sólo lo han hecho de palabra en el aula presencial.

Aspectos todos ellos lógicos y razonables en unas experiencias piloto que se inician, ilustran la complejidad que la cultura académica puede aportar al tránsito hacia un aprendizaje basado en el uso de los recursos virtuales.

\section{Epílogo: la docencia virtual como respuesta al reto de la globalidad}

Parece ser que el futuro de las instituciones formativas se sitúa en el escenario de la globalidad. Las nuevas tecnologías ofrecen la posibilidad de compartir, en un mismo escenario, las ideas y las metodologías de formación. Una visión de futuro debe centrarse en garantizar al máximo la accesibilidad a la formación de cualquier persona así como en poder garantizar modelos formativos que se adecuen a esta nueva forma de aprender.

Ferraté afirma que «las metodologías basadas en conceptos de virtualidad se irán extendiendo y generalizando a causa de las inmensas posibilidades pedagógicas y sociales que comportan. Debemos tener presente que las metodologías asociadas al concepto de virtualidad pueden ayudarnos a romper, no solamente las barreras del tiempo y del espacio sino también las barreras sensoriales» (Ferraté, 1998:187). Es cierto que la sensorialidad está presente también en la virtualidad. Y que, como hemos visto, educamos también a través de nuestra capacidad de sentir.

El nuevo paradigma educativo que configura la virtualidad no es del todo nuevo. Consideramos que su valor reside en la posibilidad que nos brinda este medio de reinterpretar, de repensar la educación y sus mecanismos. Las teorías

3. Este proyecto forma parte de uno más amplio que recibe por nombre «La Universidad Digital». 
del aprendizaje, las metodologías, la didáctica, la comunicación, etc., deben resituarse ante un espacio, el de la virtualidad, que se nos presenta abierto a todo tipo de posibilidad de creación. Un camino nuevo a explorar y que debemos tener presente para afrontar los retos formativos que se nos plantean en el nuevo milenio.

Efectivamente, los dos puntales para la constitución de este nuevo paradigma son el modelo educativo y el modelo de las organizaciones. Ambos se relacionan estrechamente y se condicionan mutuamente. Del modelo educativo hemos destacado la importancia de centrarlo en las necesidades educativas del estudiante. Quizás resulta el más evidente. Pero el más costoso, sobre todo para las organizaciones formativas superiores ${ }^{4}$, es el cambio organizativo.

Los factores en los que deberá fundamentarse la estrategia hacia la construcción de un espacio de formación virtual deben girar alrededor de los siguientes ejes:

- Accesibilidad. Garantizar el acceso a la formación de todas las personas que quieran formarse y hacerlo a través del mayor número de medios posibles. Ello comporta seguir atentamente la evolución de la tecnología.

- Modelo de aprendizaje. Trabajar en la mejora de los modelos educativos centrados en la forma de aprender. Las estrategias de aprendizaje en el escenario de un espacio de formación virtual y global no son las mismas que las usadas por los alumnos de las universidades actuales.

- Modelos de docencia. El perfil de los profesionales de la docencia deberá cambiar pasando a ser más facilitadores que instructores. Ese cambio deberá realizarse a partir de la integración en el modelo de aprendizaje compartido que conlleva el modelo educativo basado en las nuevas tecnologías.

- Estilo organizativo-cultural. Uno de los grandes retos de la globalidad es el de procurar no perder la identidad, ya no sólo cultural, sino tradicional e histórica de las organizaciones educativas que hasta ahora han funcionado y ganado prestigio. Un marco en el que no haya estilos propios de actuación docente es un espacio gris y poco atractivo. Deberemos trabajar para poner el estilo de nuestras organizaciones educativas actuales también en la red.

- Interculturalismo. La posibilidad de relacionar culturas diferentes en la red ya es una realidad que funciona. El único problema es lingüístico. No para las grandes lenguas pero sí para las lenguas minoritarias. Los traductores simultáneos ayudarán, sin duda, a la comprensión de los mensajes, pero no superarán la comprensión cultural de quien los emite o los recibe. Creemos que éste es uno de los grandes retos de la globalidad hacia la que avanzamos.

4. Consultar para uno de los posibles conceptos de universidad virtual: Richard Teare, David Davies; Eric Sandelands. The Virtual Universtity. Londres: Cassell, 1998. 
En el ámbito de las universidades empiezan a existir ya diversas modalidades. Se trata de facilitar al máximo la realización de cursos a través del web, de forma virtual, ofreciendo algunas asignaturas de forma virtual ${ }^{5}$, creando extensiones virtuales dentro de la propia institución ${ }^{6}$ o creando verdaderas universidades virtuales adosadas a la universidad presencial ${ }^{7}$. En muchos casos, la estrategia pasa por coaligarse con otras instituciones para situar los cursos virtuales en un espacio común ${ }^{8}$ o estableciendo convenios entre diferentes universidades, sean del país o continente que sean ${ }^{9}$. La organización de esta tipología de universidad virtual es simple ya que en el primer caso se reduce a la mínima configuración de una estructura virtual que permita la difusión de diferentes cursos que se realizan en distintas universidades ${ }^{10} \mathrm{o}$ bien a acuerdos políticos y de protocolos informáticos entre distintas instituciones formativas en el segundo caso ${ }^{11}$.

La realidad es que el mundo de la formación se mueve hacia el futuro con una rapidez vertiginosa. Estar en este espacio planetario de formación universitaria será una realidad en breve (de hecho ya lo es casi ahora). Como personas con nuestros valores y como institución con estilos y valores propios también tenemos la misión de garantizar que ese espacio cumpla el objetivo para el que se crea: la formación de las personas a partir del compartir conocimientos e ideas, pero desde el reconocimiento y el respeto a la diversidad.

\section{Bibliografía}

BATES, A.W. (1995). Technology, Open Learning and Distance Education. Londres: Routledge.

DUART, J.M. (1999). La organización ética de la escuela y la transmisión de valores. Barcelona: Paidós.

DuART, J.M.; SANGRÀ, A. (comp.) (2000). Aprender en la virtualidad. Barcelona: Gedisa.

5. La mayor parte de universidades españolas están haciéndolo en estos momentos.

6. Como ejemplos, sirvan la Universidad de California en Berkeley (UC Berkeley Extension) o la de Los Angeles (UCLA Online).

7. La Universidad Virtual del Instituto Tecnológico Superior de Monterrey (México) fue una de las primeras en desarrollar este modelo organizativo (http://www.ruv.itesm.mx).

8. Este es el modelo de la Western Governor's University (http://www.wgu.edu), que ha dado lugar recientemente al Governor's University System.

9. Existen ya experiencias en este sentido. La UOC ha realizado conjuntamente con la Open University de UK y la Växo Universtity de Estocolmo una experiencia de mobilidad virtual de estudiantes en el marco de un proyecto europeo (Student Virtual Mobility: SVM). El resultado fue exitoso y ha permitido desarrollar el concepto de Metacampus, promovido por la Universidad Oberta de Catalunya, y al cuál se ha unido en una experiencia piloto la UAB.

10. Sirvan como ejemplos las URL de la California Virtual University (http://www.california.edu).

11. En este sentido se celebró en diciembre de 1998 en Barcelona un Workshop del International Council for Open and Distance Education bajo el lema de «Towards the Global Virtual University Alliance». 
FERRATÉ, G. (1997). «Internet como entorno para la enseñanza a distancia». En TifFIN, J.; RAJASINGHAM, L. En busca de la clase virtual. La educación en la sociedad de la información. Barcelona: Paidós.

- (1998). «Universidad y nuevas tecnologías. El camino hacia la hiperuniversidad». En Porta, J.; LladonOSA, M. (coord.). La universidad en el cambio de siglo. Madrid: Alianza Editorial.

GuÀrdiA, L.; SANGRÀ, A. (2001). «L'Assistent de Recursos Metodològics (ARM): una eina al servei de l'elaboració de cursos». Ponencia presentada en el $V$ Debate Interuniversitario del Institut Universitari Joan Lluís Vives. Castellón.

Harasim, L.; Hiltz, S.R.; Teles, L.; Turoff, M. (1995). Learning Networks. Cambridge, MA: The MIT Press, 1995. [Existe una edición en español: Redes de aprendizaje. GEDISA-Ediuoc: Barcelona, 2000].

ICDE (1998). Towards the Global Virtual University Alliance. Report of the first Planning Workshop. Barcelona: UOC-ICDE.

LANDOW, G. (1997). Hypertext 2.0: The Convergence of Contemporary Critical Theory and Technology. Baltimore: The John Hopskins University Press.

LÉVY, P. (1999). ¿Qué es lo virtual? Barcelona: Paidós.

MOORE, M. (1989). «Editorial: Three types of interaction». American Journal of Distance Education, vol. 3, núm. 2, p. 1-6.

PETERS, O. (1989). «The iceberg has not melted: further reflections on the concept of industrialisation and distance teaching». Open Learning, vol. 4, núm. 3, p. 3-8.

RHEINGOLD, H. (1996). La comunidad virtual. Una sociedad sin fronteras. Barcelona: Gedisa.

SANGRÀ, A.; DUART, J.M. (2000). «Formación universitaria por medio de la web: un modelo integrador para el aprendizaje superior». En DUART, J.M.; SANGRÀ, A. (comp.). Aprender en la virtualidad. Barcelona: Gedisa.

Sangra, A.; Bellot, A.; Hinojosa, J. (2000). Projecte Astrolabi. 2n. Informe de l'Observatori sobre la implantació i l'ús de les Tecnologies de la Informació i la Comunicació a l'ensenyament no universitari. Barcelona: UOC. (IN3, Edu Lab, Fundació Jaume Bofill).

SCHANK, R. (1997). Virtual Learning. A revolutionari approach to building a highly skilled workforce. Nueva York: McGraw Hill.

SimOnson, M.; SCHSlOSSER, C.; Hanson, D. (1999). «Theory and Distance Education: A New Discussion». American Journal of Distance Education, vol. 13, núm. 1.

TAIT, A.; MiLls, R. (ed.) (1999). The convergence of distance and conventional education. Londres: Routdlege.

Teare, R.; Davies, D.; Sandelans, E. (1998). The Virtual University. An Action Paradigm and Process for Workplace Learning. Londres: Cassell.

WEDEMEYER, C. (1981). Learning at the Back-door. Madison: University of Wiscon$\sin$. 